\title{
Revisited fluorine abundances in the globular cluster M 22 (Research Note)
}

\author{
P. de Laverny and A. Recio-Blanco
}

\author{
Laboratoire Lagrange (UMR7293), Université de Nice Sophia Antipolis, CNRS, Observatoire de la Côte d'Azur, BP 4229, \\ 06304 Nice Cedex 4, France \\ e-mail: laverny@oca.eu
}

Received 7 July 2013 / Accepted 4 November 2013

\section{ABSTRACT}

\begin{abstract}
Aims. Fluorine is a fairly good tracer of formation histories of multiple stellar populations in globular clusters as already revealed by several studies. Large variations in fluorine abundance in red giant stars of the globular cluster M 22 have been recently reported by two different groups. Futhermore, one of these studies claims that the abundance of fluorine is anti-correlated with sodium abundances in this cluster, leading to strong conclusions on the chemical history of M 22. To validate this important finding, we re-examine the $\mathrm{F}$ abundance determinations of some of the previously studied stars.

Methods. We have reanalysed some high-resolution VLT/CRIRES spectra of RGB stars found in M 22 in order to re-estimate their fluorine abundance from the spectral synthesis of the HF line at $2.336 \mu \mathrm{m}$.

Results. Unlike what has been previously estimated, we show that only upper limits or doubtful fluorine abundances with large uncertainties in M 22 RGB stars can be derived. This is caused by an incorrect identification of continuum fluctuations as the HF signature combined with a wrong correction of the stellar radial velocity. Such continuum fluctuations could be the consequences of telluric residuals that are still present in the analysed spectra. Therefore, no definitive conclusions on the chemical pollution caused by the M 22 first stellar generation can presently be drawn from the fluorine content of this cluster.
\end{abstract}

Key words. globular clusters: individual: M 22 - stars: abundances

\section{Introduction}

Fluorine is a chemical species of special interest in studies devoted to the evolution history of stellar populations for several reasons. For instance, (i) the nucleosynthetic origin of fluorine in the Universe is still debated with two main ranges of stellar masses proposed for its production site; and (ii) fluorine abundances may help disentangle the complex formation history of the globular clusters (GCs) by looking for the chemical pollution signatures of their first stellar population. Indeed, over the last decade, several studies devoted to the abundance of fluorine in different types of galactic and extragalactic stellar populations have shed new light on their chemical history (see Recio-Blanco et al. 2012; de Laverny \& Recio-Blanco 2013, for a more detailed description).

In particular, the abundances of fluorine on the surface of some red giant branch stars (RGBs) of a few GCs have already been estimated. Most of these targeted GCs are metal-rich or have an intermediate metallicity (typically, $[\mathrm{Fe} / \mathrm{H}]<-1.2$ ). For more metal-poor clusters, only upper limits of the fluorine content of NGC $6397([\mathrm{Fe} / \mathrm{H}]=-1.99)$ and M $30([\mathrm{Fe} / \mathrm{H}]=-2.33)$ have been reported up to now (de Laverny \& Recio-Blanco 2013). Unfortunately, these too high upper limits avoid any derivation of any conclusive information on the chemical history of these clusters. It has also been shown that these $\mathrm{F}$ abundance upper limits in such metal-poor GCs are probably caused by inherent limitations of high-resolution $K$-band spectra collected with current telescopes and spectrographs.

In that figure, the only exception of fluorine abundance measurements (and not only upper limits) in metal-poor GCs is M 22 (NGC 6656) with a mean metallicity $[\mathrm{Fe} / \mathrm{H}] \sim-1.7 \mathrm{dex}$. Recently, Alves-Brito et al. (2012) have indeed reported [F/Fe] ratio estimates in five RGB stars and upper limits in two other stars of M 22. Then, D'Orazi et al. (2013, DLL13 hereafter) partly revised these abundances: their sample of six M 22 RGBs contains four stars already studied by Alves-Brito et al. (2012). DLL13 show that the telluric substraction may partly explain their different estimates of F-abundances with respect to the previous study. These new abundance estimations allowed them to derive constraints on the masses of the first-generation stars that could have chemically polluted M 22 .

However, our previous analysis of the fluorine content in metal-poor GCs (de Laverny \& Recio-Blanco 2013) has shown that F-abundances cannot be derived well in such clusters. In this research note and in to validate the F-abundances previously estimated in M 22, we thus re-examine some of the spectra collected by DLL13. We show that the fluorine content of M 22 RGB members cannot be safely estimated (see Sect. 2). This leads us to conclude in Sect. 3 that the properties of the M 22 first-generation stars (as the range of masses of the main polluters) cannot currently be drawn from the fluorine content of this cluster.

\section{Analysis of M 22 RGB CRIRES spectra around the fluorine signature}

We selected three RGB stars of the sample of DLL13 and re-analysed their spectra. We recall that these spectra were collected with the VLT/CRIRES instrument (see DLL13, for a description of the observations, their reduction and telluric features substraction). We point out that we re-analysed exactly the same spectra as DLL13 who performed the cleaning from the telluric contamination. In this note, we indeed only focus on the re-estimation of the fluorine abundances from these already reduced and cleaned spectra. 

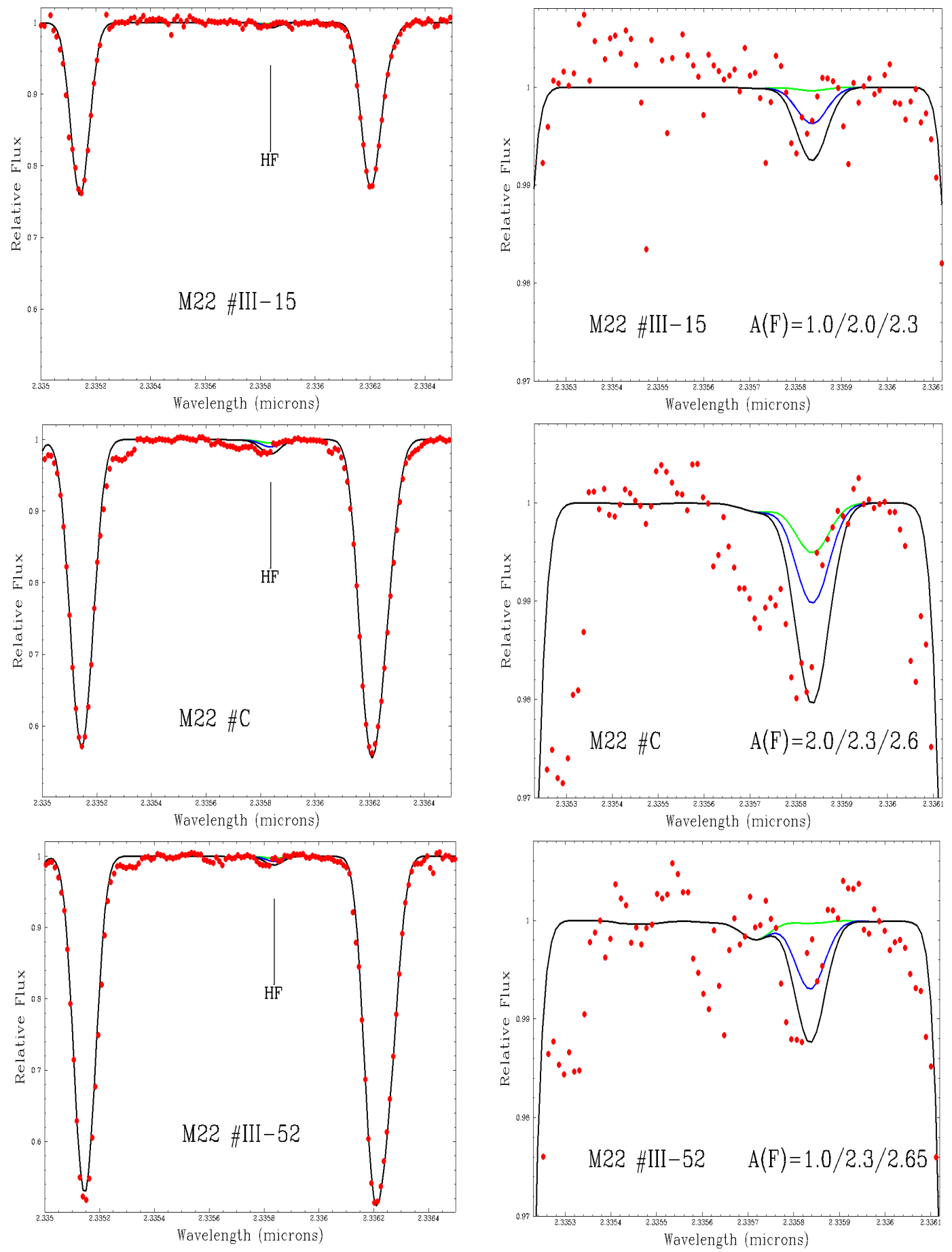

Fig. 1. Synthetic fits of the observed spectra of the M22 RGB stars \#III-15, \#C, and \#III-52 (from top to bottom). The left panels show a rather large spectral domain including the weak HF-transition together with two strong CO lines, confirming the right radial velocity correction adopted for the fits. A zoom of the left panels around the HF-line is shown in the right panels. Three different fluorine abundances spanning a wide range of possible values have been adopted for the synthetic spectra (see the labels in each right-hand panels).

The selected targets are the stars \#III-15, \#C, and \#III-52. The first one has been estimated by DLL13 to be the most fluorine-poor $([\mathrm{F} / \mathrm{Fe}]=-1.0 \mathrm{dex})$ of their sample of six M 22 RGBs, whereas the other two are proposed as quite enriched in $\mathrm{F}$ (with $[\mathrm{F} / \mathrm{Fe}]=-0.8$ and -0.6 dex, respectively). Actually, it is claimed that the star \#III-52 would be the most enriched in fluorine found so far in M 22.

The new fluorine abundances were estimated by adopting exactly the same methodology as in de Laverny \& Recio-Blanco (2013), which is actually identical to all our previous works devoted to $\mathrm{F}$ abundance determinations (see, for instance, our first paper in this series, Abia et al. 2009). We only recall that these abundance determinations are based on the synthetic modelling of the unblended HF(1-0) R9 line at $2.3358 V_{\text {micro }}$. We also adopted the same atmospheric parameters $T_{\text {eff }}, \log (g),[\mathrm{Fe} / \mathrm{H}]$, and $V_{\text {micro }}$ as DLL13. The synthetic spectra were then broadened by convolution with a Gaussian profile of $F W H M=9 \mathrm{~km} \mathrm{~s}^{-1}$ to match the observed line widths.

We show in Fig. 1 the synthetic fits of the three selected spectra around the HF transition, assuming three different fluorine 

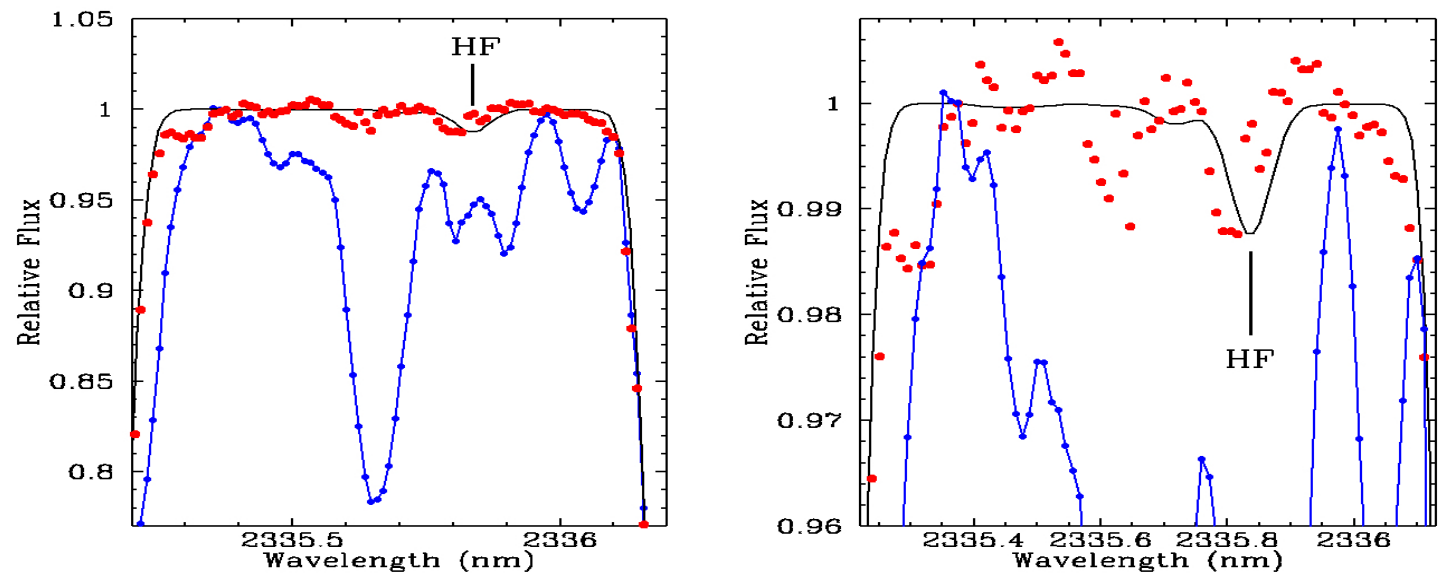

Fig. 2. Observed spectrum of the M 22 RGB star \#III-52 with (red points) and without (blue line and dots) telluric substraction, together with its corresponding synthetic fit (black line). The cleaned and synthetic \#III-52 spectra are those already shown in Fig. 1 (lower panel). The left panel is a zoom of the right one around the continuum level where the HF line should be found.

abundances spanning at least 1.3 dex. The largest of these abundances could actually be considered as an upper limit of $A(\mathrm{~F})$. From this Fig. 1, it can be clearly seen that no reliable fluorine abundances can be derived from these spectra. Indeed, if the stellar radial velocity is corrected well to match all the strong CO-lines (left panels of Fig. 1), no line feature can easily be identified at the right-hand HF-line position (see the plots in the right-hand panels of Fig. 1, showing a zoom around the HF-line). It can indeed be seen that large flux fluctuations are present around the HF line, whereas no features are predicted by the synthetic spectra in these almost perfectly line-free (continuum) regions of very metal-poor stars. Furthermore, these fluctuations have the same amplitude (a few percent) as the expected HF line in these stars. They could thus mimic the presence of the HF transition if examined over too narrow a spectral domain (see, for instance, Fig. 2 of DLL13) but, then, losing the right position for the CO lines. Therefore, for two of the stars shown in Fig. 1 (\#III-15 and \#III-52), only an upper limit of their fluorine content could be estimated safely $(A(\mathrm{~F}) \leq 2.3$ and $2.65 \mathrm{dex}$, respectively). For the RGB star \#C, if the feature seen close to the HF-line position were real (although not exactly at the right wavelength), it could only be estimated that its F-content were found in a wide range [2.0-2.6]. However, we again point out that we cannot exclude that no HF-line signature at all is present in these three spectra characterized by continuum flux fluctuations at almost all wavelengths and with similar amplitudes as the supposed HF feature.

On the other hand, we think that these flux variations could be caused by the telluric contamination that cannot be perfectly cleaned in such spectra. Even with an optimized procedure to remove the telluric absorption lines, unfortunately for a cluster like M 22, the main telluric feature that contaminates the spectra in that spectral range has a wing that can still be strong around the HF position. Owing to possible limitations on the stability of CRIRES and/or the earth atmosphere, subtracting the two spectra (the RGB target and the telluric standard, i.e. a hot star) could artificially produce residuals with almost the same amplitude (1\% to $2 \%$ ) and position as the HF-transition. Then, this could lead to possible incorrect identifications of the faintest stellar features. Actually, this possible explanation is reinforced by examining Fig. 2 where we compare the spectra of the star \#III-52 with and without telluric correction. We recall that this star is the F-richest of the DDL sample. It can be seen that the telluric absorption lines are large in this spectral domain and that all the continuum fluctuations seen in the cleaned \#III-52 spectrum are located at the telluric absorptions. In particular, because of its radial velocity, the studied HF transition is located at a telluric line that absorbs $\sim 7 \%$ of the continuum flux. After telluric cleaning, several continuum fluctuations are still present. In particular, a continuum fluctuation can be seen with an amplitude as large as the expected $\mathrm{HF}$ line $(\sim 1 \%)$ but shifted by two to three pixels with respect to the right HF-line wavelength. This artefact could thus mimic an HF-line if the stellar radial velocity is not perfectly estimated with the neighbouring $\mathrm{CO}$ lines (see Fig. 1), and then corrected. Furthermore, this Fig. 2 can be compared to the Fig. 7 of DLL13 for the star \#III-15 that has been proposed to be the most fluorine-poor of M 22 by these authors. It can be seen in their figure (lower panel) that the telluric contribution is almost null at the HF-line position, because of the \#III-15 radial velocity. In this optimal case, one can note that DLL derive their lowest fluorine abundance, unlike their F-rich star \#III-52 in which the telluric contribution is much greater and, as a consequence, much more difficult to clean.

\section{Conclusion}

We have re-examined the spectra of three M 22 RGB stars already studied by DLL13. We showed that the presence of any HF-line signature is doubtful in these spectra and that, if any of these signatures were real, only high upper limits or a wide range of possible fluorine contents in M 22 RGB stars could be estimated. We also proposed that previous estimates of the fluorine content in this cluster are probably based on incorrect identification of the HF-transition, owing to (i) the presence of continuum fluctuations (probably caused by telluric residuals) that are as large as the sought HF line; and (ii) not enough accurate radial velocity corrections. Indeed, if the radial velocity is determined well using the neighbouring CO lines, no HF signatures are seen in these spectra, leading to a totally unknown fluorine content in this cluster.

Therefore, since the three selected targets reanalysed in this note span the whole range of fluorine abundances derived by DLL13, it can be concluded that no reliable information on the fluorine content in these M 22 RGB stars can be derived from the available spectra. It is thus impossible to claim that there is any fluorine-oxygen correlation and fluorine-sodium 
A\&A 560, A74 (2013)

anti-correlation detected in M 22. This avoids any conclusion on any properties of the M 22 first-generation stars, such as the range of stellar masses of the probable main polluters.

Acknowledgements. We acknowledge the anonymous referee for comments that helped improve this work. We sincerely thank V. D'Orazi for providing the reduced and cleaned CRIRES spectra of the M 22 stars reanalysed in this note. $\mathrm{R}$. Gratton is also thanked for his constructive remarks on the manuscript.

\section{References}

Abia, C., Recio-Blanco, A., de Laverny, P., et al. 2009, ApJ, 694, 971 Alves-Brito, A., Yong, D., Meléndez, J., Vásquez, S., \& Karakas, A. I. 2012, A\&A, 540, A3

de Laverny, P., \& Recio-Blanco, A. 2013, A\&A, 555, A121

D’Orazi, V., Lucatello, S., Lugaro, M., et al. 2013, ApJ, 763, 22

Recio-Blanco, A., de Laverny, P., Worley, C., et al. 2012, A\&A, 538, A117 